\title{
Allogeneic AML Antigen-expressing Dendritic Cell Vaccine
}

National Cancer Institute

\section{Source}

National Cancer Institute. Allogeneic AML Antigen-expressing Dendritic Cell Vaccine. NCI

Thesaurus. Code C97265.

A cancer vaccine consisting of allogeneic, immortalized dendritic precursor cells derived from a patient with acute myelogenous leukemia (AML), with potential immunostimulatory and antineoplastic activities. Upon ex vivo stimulation and expansion of the precursor cells into mature, fully functional dendritic cells (DCs) and subsequent administration, the allogeneic AML antigen-expressing DC vaccine may elicit a potent cytotoxic T-cell (CTL) and antibody response against AML antigen-expressing cells, resulting in tumor cell death. 